\title{
НОВЫЙ МЕТОД УПРОЧНЕНИЯ ЛЕДОВЫХ МАССИВОВ, ФОРМИРУЕМЫХ В УСЛОВИЯХ ЕСТЕСТВЕННОГО ХОЛОДА
}

\author{
Г.Ю. Гончарова ${ }^{1,2}$, Р.О. Степанов ${ }^{1}$, Т.С. Разомасова ${ }^{1}$, И.А. Королев ${ }^{2}$, \\ Д.О. Туралин ${ }^{1}$ Ю.А. Кулагин ${ }^{3}$ Ю.Г. Паршиков ${ }^{4}$
}

${ }^{1}$ МГТУ им. Н.Э. Баумана, 2-я Бауманская ул., 5 стр.1, Москва, Российская Федерация, 105005 E-mail: galinagoncharova@mail.ru, stepanovr@bmstu.ru, trazomasova@yandex.ru_denis.turalin@gmail.com ${ }^{2}$ Всероссийский Научно-исследовательский институт холодильной промышленности - филиал ФБГНУ «ФНЦ пищевых систем им. В.М. Горбатова» РАН, ул. Костякова, 12, Москва, Российская Федерация, 127422

E-mail: gigja@yandex.ru

${ }^{3}$ Государственный научный центр Российской Федерации Федеральное государственное унитарное предприятие «Центральный научно-исследовательский институт химии и механики» (ГНЦ РФ ФГУП «ЦНИИХМ»), ул. Нагатинская, 16а, Москва, Российская Федерация, 115487

E-mail: kulaginyua@mail.ru

${ }^{4}$ Федеральное государственное бюджетное учреждение науки Межведомственный центр аналитических исследований в области физики, химии и биологии при Президиуме Российской академии наук (МЦАИ РАН), ул. Профсоюзная, 65 с.6, Москва, Российская Федерация, 117342

E-mail: parshikov62@ rambler.ru

С освоением Арктических территорий особую актуальность приобретает использование льда в качестве строчтельного материала при создании автозимников, ледовых переправ, взлетно-посадочных полос и строений. Главными сдерживающиими факторами его применения является хрупкость и низкая прочность ледовой матрицы. Внесение в состав льда модифицирующих соединений, позволяет повысить его прочность более чем в 1,5 раза. Ключевой проблемой технологии формирования модифицированного льда является необходимость создания и поддержания оптимальной концентрации модификаторов (в диапазоне $\xi_{\text {мод }}=150-300$ pрm) в наиболее механически нагруженной зоне массива. В отличие ото льда, формируемого при заливке крытых катков и движении фронта кристаллизации снизу-вверх, при формировании ледовых массивов в естественных условиях распределение модификаторов имеет принципиально иной характер, исследование которого и являлось главной целью представленной статьи. Виервые методом атомно-эмиссионной спектроскопии авторами получены экспериментальные данные об эпюре распределения модифицирующих соединений по глубине массива льда, сформированного за счет естественного холода. Циклический характер экспериментально полученной кривой подтвердил возможность послойного «запирания» вносимых химических соединений внутри массива при отводе тепла к окружающему воздуху и движении фронта кристаллизации сверху вниз. В указанных условиях основными факторами, позволяющими направленно изменять концентрацию вносимых модификаторов с целью повыщения общей несущей способности ледового массива, могут быть толщина заливаемого слоя и продолюсительность перерывов между заливками. Показана высокая перспективность дальнейших исследований с учетом характерных для Арктики температурных условий.

Ключевые слова: химическое модифицирование, лед, естественный холод, фронт кристаллизации, прочностные свойства, эпюра распределения

Для цитирования:

Гончарова Г.Ю., Степанов Р.О., Разомасова Т.С., Королев И.А., Туралин Д.О., Кулагин Ю.А., Паршиков Ю.Г. Новый метод упрочнения ледовых массивов, формируемых в условиях естественного холода. Рос. хим. ж. (Ж. Рос. хим. об-ва). 2021. T. LXV. № 3. С. 33-41

For citation:

Goncharova G.Yu., Stepanov R.O., Razomasova T.S., Korolev I.A., Turalin D.O., Kulagin Yu.A., Parshikov Yu.G. A New method of an ice blocks formed under a natural cooling condition strengthening. Ros. Khim. Zh. 2021. V. 65. N 3. P. 33-41 


\title{
A NEW METHOD OF AN ICE BLOCKS FORMED UNDER A NATURAL COOLING CONDITION STRENGTHENING
}

\author{
G.Yu. Goncharova ${ }^{1,2}$, R.O. Stepanov ${ }^{1}$, T.S. Razomasova ${ }^{1}$, I.A. Korolev ${ }^{2}$, \\ D.O. Turalin ${ }^{1}$, Yu.A. Kulagin ${ }^{3}$, Yu.G. Parshikov ${ }^{4}$
}

\begin{abstract}
${ }^{1}$ Bauman Moscow State Technical University, 2-ya Baumanskayast., 5/1, Moscow, 105005, Russia
E-mail: galinagoncharova@mail.ru, stepanovr@bmstu.ru, trazomasova@yandex.ru,denis.turalin@gmail.com ${ }^{2}$ All-Russian Research Institute of the Refrigeration Industry-branch of the FBGNU "V. M. Gorbatov Federal Research Center for Food Systems" of the Russian Academy of Sciences, Kostyakovast., 12, Moscow, Russian Federation, 127422

E-mail: gigja@yandex.ru

${ }^{3}$ The Central Scientific Research Institute of Chemistry and Mechanics, Nagatinskaya str., 16a, Moscow, Russian Federation, 115487

E-mail: kulaginyua@mail.ru

${ }^{4}$ Federal State State-financed Institution of Sciences Interdepartmental Center of Analytic Studies in the Sphere of Physics, Chemistry and Biology under Presidium of Russian Academy of Sciences (ICAS RAS), 65 Trade Union Street, p. 6, Moscow, Russian Federation, 117342

E-mail: parshikov62@ rambler.ru
\end{abstract}

With the Arctic territories exploration, the use of ice as a building material for building of a winter roads, ice crossings, airstrips and buildings becomes especially relevant. The main limiting factors of its use are the fragile and low strength of the ice matrix. The application of modifying compounds in the ice composition makes it possible to increase its strength by more than 1.5 times. The key problem of the modified material forming technology is the need to create and maintain an optimal concentration of modifiers (in the range $\xi_{\text {mod }}=150-300$ ppm) in the mechanically most loaded zone of the solid. Unlike artificial ice rinks ice, where the crystallization front moves from bottom to top, during the formation of ice solids in natural conditions, the distribution of modifiers in depth has a fundamentally different character, the study of which is the main task of the present article. For the first time, by a method of atomic emission spectroscopy the experimental data on the distribution of modifying compounds over a depth of an ice block formed under a natural cooling condition were obtained. The cyclical form of the experimentally obtained curve confirmed the possibility of layer-by-layer "blocking" of the dissolved chemical compounds inside the ice block during heat removal to the environmental cold air and the movement of the crystallization front from top to bottom. Under these conditions, the main factors that make it possible to purposefully shift the concentration of the ice modifiers in order to increase the total bearing capacity of the ice solid may be the thickness of the poured layers and the duration of the breaks between pours. A further research seems particularly promising, taking into account the Arctic temperature conditions.

Key words: chemical modification, ice, natural cooling, crystallization front, strength properties, concentration diagram

\section{ВВЕДЕНИЕ}

Россия занимает первое место в мире по площади северных и арктических территорий $[1,2]$. Неисчерпаемые запасы снега и естественного холода издревле использовались для сооружения природных холодильников, строений, зимников, водных причалов и переправ [3-5]. Однако использованию водного льда в качестве полноценного конструкционного материала всегда препятствовала высокая хрупкость и низкая прочность ледовой матрицы [6-10]. Особую проблему это приобретает при строительстве водных переправ и конструкций, характеризующихся наличием напряжений растяжения, что крайне нежелательно для хрупких материалов [11].

Современные методы упрочнения искусственного льда спортивных сооружений основаны 
на внесении в заливаемую воду микродоз полимеров (ледовых модификаторов) [12, 13]. Модификаторы, дислоцирующиеся в межкристаллическом пространстве, выступают в роли демпфера и значительно снижают интенсивность межкристаллических взаимодействий [14-16]. Согласно проведенным нами ранее экспериментам внесение модификаторов позволяет более чем в 2 раза повышать прочность ледового массива (рис. 1, а). Зависимость максимального напряжения при испытании образцов на изгиб от суммарной концентрации вносимых соединений имеет экстремум. Оптимальные значения концентраций, обеспечивающие максимальное повышение прочностных свойств, находятся в диапазоне $\xi_{\text {мод }}=150-300$ ppm. С дальнейшим увеличением концентрации модифицирующих веществ их эффективность уменьшается, и при $\xi_{\text {мод }}=1000$ ppm происходит снижение прочности ледового массива до исходного уровня прочности. Если концентрация модификаторов превышает 10000 ppm (1\%), то прочность ледовой матрицы оказывается на 10-15\% ниже, чем прочность ледового массива из дистиллированной воды. Кроме того, добавление модификаторов позволяет увеличить значение максимально допустимой деформации испытываемых образцов перед разрушением более чем на $50 \%$ (рис. 1, б).

Ключевой задачей технологии заливки спортивных покрытий является создание и поддержание оптимальной концентрации модификаторов в механически нагруженной поверхности массива льда в течение всего срока эксплуатации покрытия. При этом для ледовых полей с искусственным хладоснабжением вся теплота отводится вниз, к циркулирующему в бетонном основании хладоносителю, а фронт кристаллизации заливаемых водных растворов движется снизу-вверх, вытесняя все вводимые модификаторы к поверхности ледяного массива [12].

При формировании массива льда в естественных условиях за счет отвода теплоты к холодному воздуху фронт кристаллизации заливаемых водных растворов движется сверху вниз, «запирая» внутри формируемого массива внесённые модификаторы, поэтому следует ожидать, что распределение вносимых соединений в этом случае будет иметь принципиально иной характер. Учитывая, что именно наличие модифицирующих соединений снижает хрупкость ледовой матрицы, интерес представляет характер их распределения по глубине (и зонам механических напряжений) при отводе тепла к окружающему воздуху, что и является основной целью представленной статьи. Кроме этого, необходимо было определить факторы, влияющие на эпюру распределения и возможность направленного изменения дислокации модифицированных слоёв льда, их толщины и концентрации. Для этого был разработан экспериментальный стенд и соответствующая методика проведения эксперимента и обработки результатов.

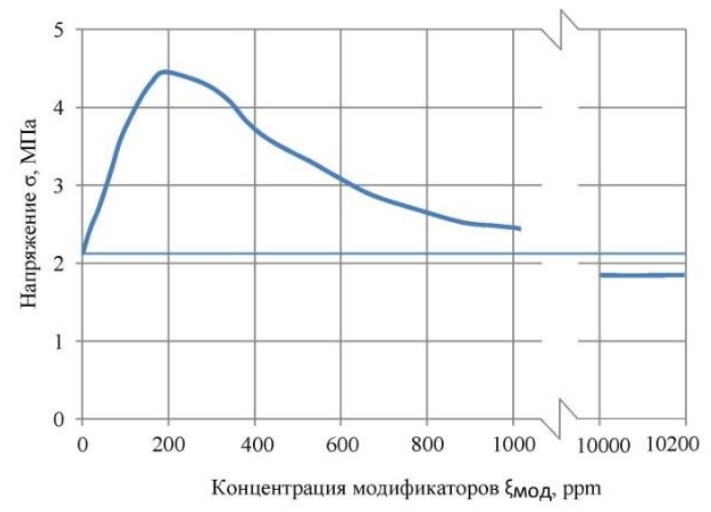

a)

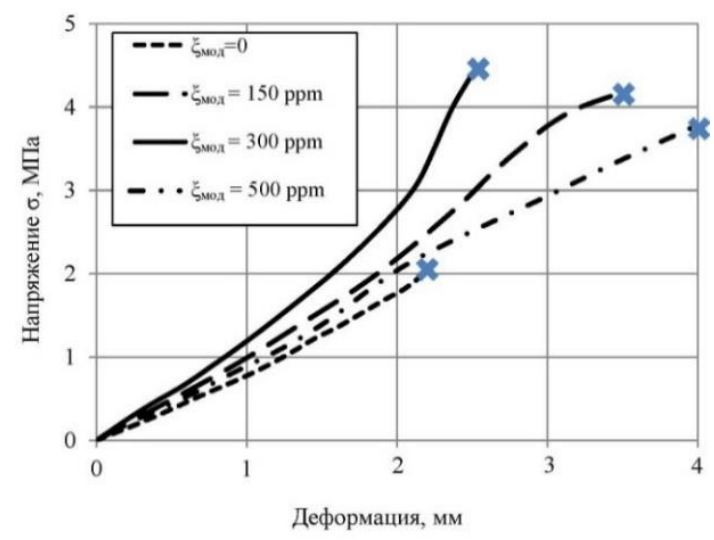

б)

Рис. 1. Влияние модификаторов на прочность льда а) Зависимость максимально допустимого напряжения в образце от концентрации модификаторов; б) кривые нагруже-

ния при испытании ледовых образцов на прочность

\section{ЭКСПЕРИМЕНТАЛЬНЫЙ СТЕНД И МЕТОДИКА ПРОВЕДЕНИЯ ЭКСПЕРИМЕНТА}

Внутри холодильной камеры с установленной температурой воздуха $t_{\mathrm{B}}=-15 \pm 0,5^{\circ} \mathrm{C}$ была размещена теплоизолированная снизу и по бокам опалубка из пенополистирола с внутренними размерами $\mathrm{W}=450 \mathrm{мm}, \mathrm{L}=950$ мм. Установленный внутри камеры вентилятор с частотным регулированием обеспечивал вынужденное движение воздуха со скоростью $\mathrm{v}_{\mathrm{B}}=0,5 \mathrm{M} / \mathrm{c}$. 
Формирование ледового массива внутри опалубки осуществлялось следующим образом. Первоначально была наморожена ледовая подоснова суммарной толщиной $\delta=13$ мм, имитирующая естественный лёд в арктической зоне. Для этого были выполнены пять последовательных заливок дистиллированной воды с толщиной слоя $\delta_{\text {дист }}=2,6$ мм. После заливки каждого из слоев осуществлялась временная выдержка до полного замерзания слоя. Контроль за температурой поверхности ледового массива перед очередной заливкой осуществлялся с помощью контактного термометра «Testo 0560-1109», с погрешностью, не превышающей $\Delta \mathrm{t}_{\text {пов }}= \pm 1^{\circ} \mathrm{C}$. Далее намораживание ледового массива производилось по схеме $(1+2)$, то есть последовательным чередованием заливок одного слоя водного раствора модификаторов с концентрацией $\xi=300 \mathrm{ppm}$ и температурой $\mathrm{t}_{\text {мод }}=55^{\circ} \mathrm{C}$ и двух слоев дистиллированной воды с температурой $\mathrm{t}_{\text {дист }}=15^{\circ} \mathrm{C}$. По этой схеме суммарная высота ледового массива внутри опалубки была доведена до $\mathrm{H}=100$ мм. В состав вносимой смеси входили три группы модифицирующих соединений: мелкодисперсная стабилизированная суспензия политетрафторэтилена (ПТФЭ), микроэмульсия кремнийорганических соединений, а также кремнийорганическое масло в долевом соотношении концентрацией в водном растворе, соответственно, 129/129/42 ppm.

После завершения формирования ледового массива было произведено его механическое расслаивание. Толщина каждого снимаемого слоя составляла $\delta=2$ мм. Далее производилось раздельное расплавление стружки каждого слоя и сбор раствора для анализа концентрации содержащихся в нем модификаторов.

Концентрация модификаторов в слоях ледового массива определялась по содержанию кремния(Si) методом атомно-эмиссионной спектроскопии с индуктивно-связанной плазмой и блоком ультразвукового диспергирования с 10 кратным разбавлением на спектрометре «Shimadzu ICPE 9820». При этом наличие двух видов обзора (аксиальный/радиальный) позволяет определять наличие элементов в широком диапазоне концентраций, вплоть до ppb.

\section{МАТЕМАТИЧЕСКАЯ МОДЕЛЬ}

Распределение вносимых модифицирующих соединений в ледовом массиве, формируемом за счет естественного холода, определяется двумя основными механизмами. Во-первых, при разливе каждого последующего слоя воды происходит подплавление льда, и в результате смешения воды и расплава образуется некоторая промежуточная концентрация, определяемая температурой, исходной концентрацией модификаторов в разливаемой воде и, соответственно, глубиной подплавленного слоя льда. По мере наращивания ледового массива включается также второй механизм, обусловливающий перераспределение внесённых модифицирующих соединений под действием термической и концентрационной составляющих диффузии. Ранее [12] на основании фундаментальных уравнений, описывающих диффузионные процессы, и результатов собственных исследований авторами была предложена сотовокапиллярная модель перераспределения вносимых модифицирующих соединений. Исследования, в том числе методом декорирования, показали, что межкристаллическое пространство, в котором преимущественно дислоцируются модифицирующие соединения, выполняет функцию «транспортных путей», по которым происходит их последующее перераспределения под действием обеих движущих сил диффузии. [12, 13]. Однако возможность численного моделирования этого процесса существенно ограничена, прежде всего, тем, что вносимая смесь модификаторов не является единой диффундирующей средой, а входящие в состав смеси компоненты различаются по молекулярной массе до шести порядков а.е.м. (от $10^{3}$ до $10^{9}$ а.е.м.), обладают различной вязкостью, поверхностной активностью и т.д. Кроме этого, достоверное математическое описание конфигурации межкристаллических «транспортных путей» также не представляется возможным. Поэтому при математическом моделировании учитывался только первый механизм формирования эпюры распределения по глубине массива - смешение. Затем расчётная эпюра сравнивалась с экспериментальными данными, а расхождение полученных эпюр в первом приближении может характеризовать вклад второго (диффузионного) механизма в реальное распределение модифицирующей смеси по глубине массива.

В рамках описанного подхода выполним моделирование процесса намораживания слоев льда с использованием метода контрольного объ- 
ема на основании баланса теплоты [17]. Вновь заливаемый слой воды толщиной $\delta_{i}^{80 д}, \mathrm{M}$, с концентрацией модификаторов $\xi_{i}^{в о д}, \mathrm{ppm}$, и температурой

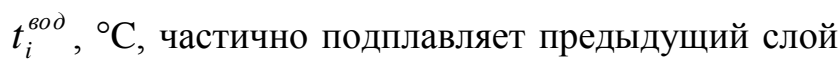
льда на величину $\delta_{i}^{n л}$, мм:

$$
\delta_{i}^{n л}=\frac{\delta_{i}^{\text {вод }} \cdot C p_{\text {вод }} \cdot\left(t_{i}^{\text {вод } \left.-t_{\phi n}\right)}\right.}{\Delta H_{\phi n}},
$$

где $C p_{\text {вод }}=4,186 \kappa Д ж /(\kappa 2 \cdot K)$ - удельная изобарная теплоемкость воды; $t_{\phi n}=0{ }^{\circ} \mathrm{C}-$ криоскопическая температура раствора; $\Delta H_{\phi n}=334$ кДж/кг энтальпия плавления льда. В результате плавления и смешения образуется слой $\delta_{i}^{\text {смеш }}$ воды со средней концентрацией модификаторов $\xi_{i}^{c м е м}, \mathrm{ppm}$, которая может быть рассчитана по соотношению:

$$
\xi_{i}^{\text {смеи }}=\frac{\delta_{i}^{\text {вод }} \cdot \xi_{i}^{\text {вод }}+\int_{\delta_{i}^{\text {mel }}} \xi(\delta) \cdot d \delta}{\delta_{i}^{6 о д}+\delta_{i}^{n л}}=\frac{\delta_{i}^{\text {вод }} \cdot \xi_{i}^{\text {вод }}+\xi_{i}^{n л} \cdot \delta_{i}^{л л}}{\delta_{i}^{6 о д}+\delta_{i}^{n л}} .
$$

За счет отвода тепла слой водного раствора переходит в твердое состояние с концентрацией модификаторов, распределенных по некоторой неизвестной зависимости, определяемой, в первую очередь, скоростью движения фронта фазового превращения и составом смеси используемых модификаторов. В качестве допущения примем, что распределение в замерзающем слое описывается полиномиальной функцией вида $\xi(\delta)=a \cdot \xi_{i}^{\text {смеи }}+c_{i} \cdot \delta^{n}$, где параметры $a$ и $n$ позволяют задать предполагаемую форму распределения. Коэффициент $c_{i}=\xi_{i}^{\text {смеш }} \cdot(1-a) \cdot(n+1) \cdot\left(\delta_{i}^{\text {смеш }}\right)^{-n}$ обеспечивает выполнение закона сохранения вещества (равенство площадей в координатах х- $)$ ). При этом, чем выше скорость движения фронта, тем ближе распределение модификаторов к исходному: $a \rightarrow 1$, $c_{i} \rightarrow 0$. В случае предельно медленного протекания процесса фазового перехода $a \rightarrow 0$ и наблюдается вытеснение модификаторов в направлении движения фронта фазового перехода.

Отдельно рассмотрим вопрос расчета остаточной средней концентрации $\xi_{i}^{o c m}$ в i-ом слое $\delta_{i}=\delta_{i}^{\text {смеш }}-\delta_{i+1}^{n л}$ с учетом заливки сверху слоя

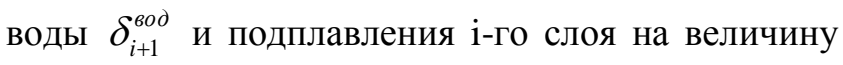
$\delta_{i+1}^{n л}$. Для случаев движения фронта фазового превращения вниз $\xi_{i}^{n л_{-} в н и з ~ и ~ в в е р х ~} \xi_{i}^{n л_{-} в в е р x}$ соответственно меняется направление оси координат в функции концентрации $\xi(\delta)$ внутри контрольного объема:

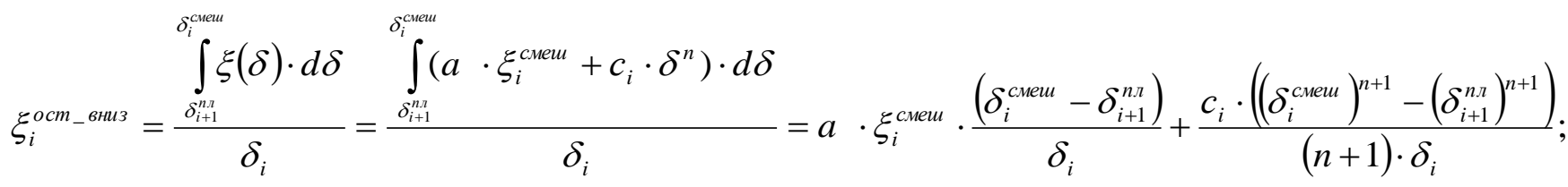

$$
\begin{aligned}
& \xi_{i}^{\text {ост }}{ }_{- \text {вер } x}=\frac{\int_{0}^{\delta_{i}} \xi(\delta) \cdot d \delta}{\delta_{i}}=\frac{\int_{0}^{\delta_{i}}\left(a \cdot \xi_{i}^{\text {смеш }}+c_{i} \cdot \delta^{n}\right) \cdot d \delta}{\delta_{i}}=a_{i} \cdot \xi_{i}^{\text {смеш }}+\frac{c_{i} \cdot \delta_{i}^{n}}{n+1} .
\end{aligned}
$$

Средняя концентрация подплавленной части i-го слоя $\delta_{i+1}^{n \pi}$ при заливке $(\mathrm{i}+1)$-го слоя водного раствора модификаторов, соответственно, для случаев движения фронта фазового превращения в лед вниз $\xi_{i+1}^{n л_{-} в н и з ~}$ и $\xi_{i+1}^{n л_{-} в в е р x}$ вверх:

$$
\begin{aligned}
& \xi_{i+1}^{n л_{-} \text {вниз }}=\frac{\int_{0}^{\delta_{i+1}^{n л}}\left(a \cdot \xi_{i}^{\text {смеш }}+c_{i} \cdot \delta^{n}\right) \cdot d \delta}{\delta_{i+1}^{n л}}=a \cdot \xi_{i}^{\text {смеш }}+\frac{c_{i} \cdot\left(\delta_{i+1}^{n \pi}\right)^{n}}{n+1}
\end{aligned}
$$

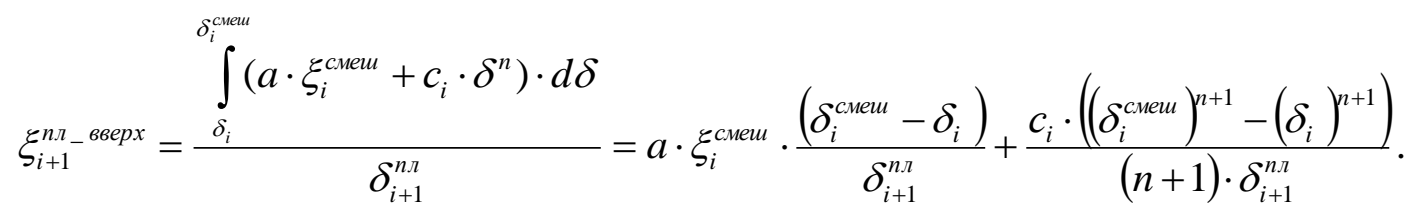




\section{ОБСУЖДЕНИЕ РЕЗУЛЬТАТОВ}

Для определения величин коэффициентов полинома а и $\mathrm{n}$ в предложенной функции $\xi(\delta)=a \cdot \xi_{i}^{\text {смеш }}+c_{i} \cdot \delta^{n}$, описывающей распределение концентраций в замерзающем слое, были проведены расчеты распределения концентраций модификаторов по глубине массива при движении фазового перехода снизу вверх и их сравнение с полученными авторами ранее экспериментальными данными [12] (рис. 2). В этом случае заливка осуществлялась по схеме чередующихся слоёв $(1+1)$ : один слой дистиллированной воды - один слой с модификаторами, температура заливаемой воды составляла отплюс 55 до плюс $65^{\circ} \mathrm{C}$. Можно видеть, что у поверхности ледового массива концентрация модифицирующих веществ $\xi_{\text {мод }}$ существенно превышает их концентрацию в глубине массива. Это обусловлено тем, что большая часть внесённых соединений, обладающих высокой поверхностной активностью, вытеснялась фронтом кристаллизации к поверхности ледового массива. Прослеживается также значительное влияние скорости кристаллизации на характер распределения внесенных соединений. При малых скоростях кристаллизации (температурах хладоносителя $\mathrm{t}_{\mathrm{xл}} \approx-8^{\circ} \mathrm{C}$ ) значения концентрации полимеров в поверхностном слое в 15 раз превышает их содержание во внутренних слоях - сплошная линия и квадратные маркеры (рис. 2). С увеличением скорости кристаллизации (температурах хладоносителя $\mathrm{t}_{\text {хл }} \approx-12{ }^{\circ} \mathrm{C}$ и $-16{ }^{\circ} \mathrm{C}$ ) отношение концентраций на поверхности и глубине снижается до 6 и 2 раз, соответственно.

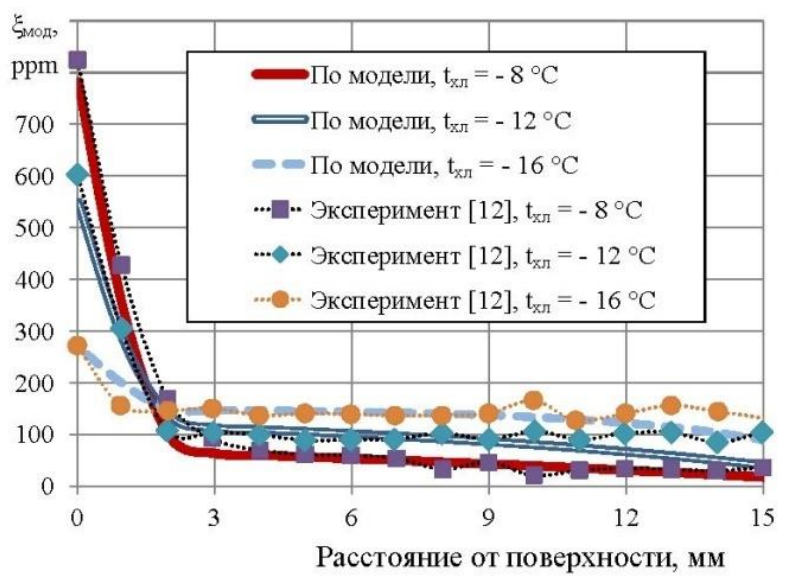

Рис. 2. Распределение концентрации модификаторов по глубине ледового катка при различных температурах хладоносителя в процессе заливки $\left(\mathrm{t}_{\mathrm{xл}}=-8 ;-12 ;-16^{\circ} \mathrm{C}\right)$

сравнение результатов расчетов по модели с экспериментальными данными
Варьирование параметров полинома $a$ и $n$ для трех различных скоростей движения фронта фазового превращения позволило определить коэффициенты полинома, обеспечивающие наилучшее приближение расчётных кривых к экспериментальным данным: $\mathrm{a}=0 ; \mathrm{n}=2 ; 4,5$ и 5,5 , соответственно для $\mathrm{t}_{\mathrm{xл}}=-8 ;-12 ;-16{ }^{\circ} \mathrm{C}$. Предложенный подход к моделированию позволяет адекватно описать распределение модификаторов по толщине ледового массива.

Результаты расчетов распределения концентрации модификаторов в массиве, формируемом в условиях естественного холода, в сравнении с экспериментальными данными, полученными на атомно-эмиссионном спектрометре, показаны на рисунке 3. Эти результаты подтверждают принципиально иное в сравнении с ледовыми катками распределение модификаторов по глубине массива.

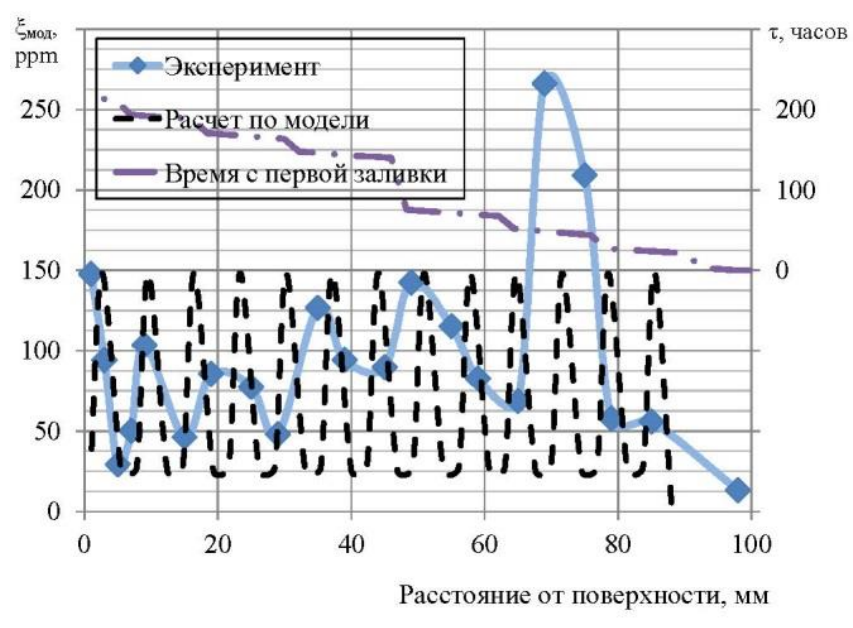

Рис. 3. Распределение концентрации модификаторов по глубине ледового массива, формируемого в условиях естественного холода при температуре воздуха $\left(\mathrm{t}_{\mathrm{B}}=-15^{\circ} \mathrm{C}\right)$ : сравнение результатов расчетов по модели с экспериментальными данными

С физической точки зрения замораживание сверху вниз каждого последующего заливаемого слоя должно способствовать «запиранию модифицирующих соединений и зигзагообразному их распределению по толщине массива, что подтверждается результатами вычислений по расчетной модели - пунктирная линия на рис. 3. Однако расчётная модель учитывает только эффект смешения концентраций при заливке каждого нового и подплавлении предыдущего слоя льда (как чистого, так и с модификаторами). При этом в перераспределение внесенных соединений включается описанный ранее механизм термической и концентрационной составляющих диффузии, действующий 
не только в периоды заливки и застывания слоя льда, а в течение всего времени формирования и последующей эксплуатации ледового массива. Концентрационная диффузия модификаторов из зон с более высокой концентрацией в обе стороны от насыщенных слоёв и термодиффузия переменного направления (в периоды заливок и длительного ночного выдерживания массива) способствуют постепенному сглаживанию пиков - голубая линия на рис. 3 .

Циклический характер экспериментально полученной кривой подтвердил возможность послойного «запирания» вносимых химических соединений внутри массива при отводе тепла к окружающему воздуху и движении фронта кристаллизации сверху вниз. Внимания заслуживает локальный экстремум концентрации в начальной стадии формирования массива, расположенный между 25 - 35 мм от его основания. По всей видимости, он может объясняться длительным перерывом (выходными днями) и полным промораживанием массива до температуры в камере равной $-15^{\circ} \mathrm{C}$. Кристаллизация слоёв, разлитых непосредственно после перерыва, происходила с существенно большей скоростью, чем при периодических заливках, производимых на непереохлаждённый массив. Таким образом, было показано, что основными факторами, позволяющими направленно изменять концентрацию вносимых модификаторов во вновь образуемом слое, могут быть температура воздуха в камере и степень охлаждения предыдущего слоя льда.

Для подтверждения возможности разнонаправленного действия температурной составляющей диффузии авторами было численно смоделировано и проанализировано (с использованием ПО с открытым исходным кодом «OpenFoam») нестационарное распределение температур в ледовом массиве при достижении толщины $\delta_{\text {л }}=70$ мм в процессе последующих заливок слоев жидкости $\delta_{\text {ж }}=2,3$ мм с температурой $15^{\circ} \mathrm{C}$. На нижней и верхней поверхности ледового массива с исходной температурой $\mathrm{t}_{л}=$ минус $15^{\circ} \mathrm{C}$ (после ночного перерыва) были приняты соответствующие граничные условия: тепловой поток $\mathrm{q}_{\text {осн }}=0$ Вт/ $\mathrm{M}^{2}$ со стороны теплоизолированного основания и коэффициент теплоотдачи воздуха $\alpha_{\mathrm{B}}=15 \mathrm{BT} /\left(\mathrm{M}^{2} \cdot \mathrm{C}^{\circ}\right)$, при температуре воздуха $t_{\mathrm{B}}=-15^{\circ} \mathrm{C}$ с верхней стороны массива. После первой заливки наблюдается существенное повышение температуры по всей толщине ледового массива, у основания он прогревается до $\mathrm{t}_{\mathrm{ocн}}=-8^{\circ} \mathrm{C}$ (рис. 4 , кривая 4). После полного замерзания первого слоя и осуществления второй заливки (кривая 5) спустя непродолжительное время в основании ледовый массив прогревается уже до $\mathrm{t}_{\text {осн }}=-1,8^{\circ} \mathrm{C}$ (рис. 4 , кривая 8). В дальнейшем, если не осуществлять дополнительных заливок, температура ледового массива постепенно в течение 8-10 ч вновь снижается до $\mathrm{t}_{\mathrm{r}}=-15^{\circ} \mathrm{C}$ (рис. 4 , кривые 9-11). Указанный процесс распространения температурных волн способствует более интенсивному переносу модифицирующих соединений механизмом термической диффузии.

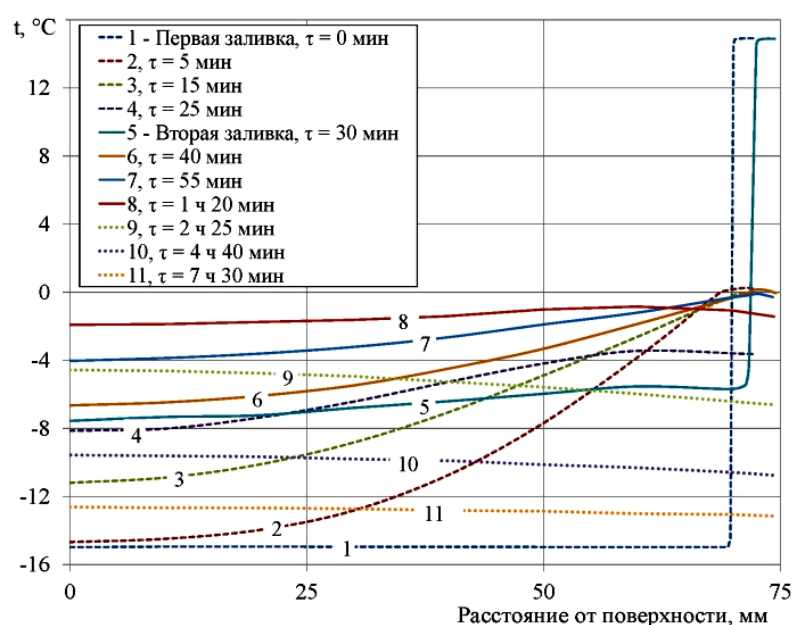

Рис. 4. Результаты расчетов нестационарного распределения температур по толщине ледового массива в процессе заливки на его поверхность слоев воды и при последующем его охлаждении

С практической точки зрения циклический характер распределения модификаторов в массивах, сформированных за счёт естественного холода, позволяет добиваться значительно большего увеличения несущей способности ледового покрытия по сравнению с ледовыми катками с искусственным хладоснабжением, на которых их концентрация монотонно возрастает к поверхности.

\section{ВЫВОДЫ}

Впервые с использованием метода атомноэмиссионной спектроскопии проведены экспериментальные исследования по изучению распределения упрочняющих модифицирующих соединений по глубине ледового массива, сформированного за счёт естественного холода.

Экспериментально подтверждена возможность послойного «запирания» вносимых химических соединений внутри массива при отводе тепла к окружающему воздуху, а циклический характер распределения модификаторов позволяет добиваться значительно большего увеличения несущей способности ледового покрытия по сравне- 
нию с ледовыми катками с искусственным хладоснабжением.

Определены факторы, позволяющие направленно изменять дислокацию по глубине массива льда слоёв повышенной концентрации внесённых соединений в соответствии с расположением наиболее нагруженных зон, например, в зоне растяжения при характерной для конструкционных материалов нагрузке на изгиб.

Разработана расчётная модель, описывающая процесс структурирования ледовых массивов с использованием естественного холода и распределения модификаторов в результате смешения разливаемой жидкости и подплавленного льда. Показано, что кроме смешения на процесс их распределения также оказывает влияние процесс термической и концентрационной диффузии, который

\section{ЛИТЕР А Т У Р А}

1. Shiklomanov N.I. From exploration to systematic investigation: development of geocryology in 19th and Early20thCentury Russia. PhysicalGeography. 2005. V. 26. N 4. P. 249-263. DOI: 10.2747/0272-3646.26.4.249.

2. Семенов С. М. и др. Методы оценки последствий изменения климата для физических и биологических систем. М.: ФГБУ Научно-исследовательский центр космической гидрометеорологии Планета. 2012. 512 с.

3. Корнилова М.А., Егоров А.Л. Снег и лед как строительные материалы. Транспортныеитранспортно-технологическиесистемы. 2014. С. 125-129.

4. Wazney L., Clark S.P., Wall A.J. Field monitoring of secondary consolidation events and ice cover progression during freeze-up on the Lower Dauphin River, Manitoba. Cold Regions Science and Technology. 2018. V. 148. P. 159-171. DOI: 10.1016/j.coldregions.2018.01.014.

5. Beltaos $S$. Freeze up jamming and formation of ice cover. In River ice Formation.Committee on River Ice Processes and the Environment, Canadian Geophysical Union, Hydrology Section: Edmonton, AB, Canada. 2013. P. 181-256.

6. Крупина Н. А. и др. Комплексные исследования процессов нарастания и механики разрушения льда естественного намерзания в большом ледовом бассейне ААНИИ. Проблемы Арктики и Антарктики. 2008. №. 2. С. 7-20.

7. Бузник В. М. и др. Физико-механические свойства композиционных материалов на основе ледяной матрицы. Материаловедение. 2017. №. 2. С. 33-40.

8. Васильев Н.К., Иванов А.А., Шаталина И.Н. Методы упрочнения и армирования льда для конструкций гидротехнических сооружений из ледяных и льдогрунтовых композитов. Сибирский журнал чистой и прикладной математики. 2013. Т. 13. №. 3. С. 31-37.

9. Herrnring H. et al. A cohesive model for ice and its verification with tensile splitting tests. 12th European LS-DYNA Conference 2019. Koblenz, Germany. 2019. 9 p.

10. Iliescu D. et al. Strengtheningicethroughcyclicloading. Journal of Glaciology. 2017. V. 63. N 240. P. 663-669. DOI: 10.1017/jog.2017.32. способствует сглаживанию концентрационных пиков и более равномерному распределению в теле льда вносимых упрочняющих соединений.

Подтверждена высокая перспективность дальнейших исследований влияния температурных условий формирования ледяных массивов, в том числе суточных и сезонных колебаний температур, а также температур характерных для арктических условий, на распределение модификаторов по глубине, а также процессов термической диффузии полимеров в межкристаллическом пространстве льда.

Исследование выполнено при финансовой поддержке РФФИ в рамках научного проекта № 20-08-00120, а также при поддержке Министерства Образования и Науки Российской Федерации.

\section{REFERENCES}

1. Shiklomanov N.I. From exploration to systematic investigation: development of geocryology in 19th and Early20thCentury Russia. PhysicalGeography. 2005. V. 26. N 4. P. 249-263. DOI: 10.2747/0272-3646.26.4.249.

2. Semenov S.M. et al. Methods for assessing the effects of climate change for physical and biological systems. M.: FGBU Research Center of Space Hydrometeorology Planeta, 2012. 512 p. (in Russian).

3. Kornilova M.A., Egorov A.L. Snow and ice as building materials. Transport and transport-technological systems. 2014. P. 125-129. (in Russian).

4. Wazney L., Clark S.P., Wall A.J. Field monitoring of secondary consolidation events and ice cover progression during freeze-up on the Lower Dauphin River, Manitoba. Cold Regions Science and Technology. 2018. V. 148. P. 159-171. DOI: 10.1016/j.coldregions.2018.01.014.

5. Beltaos $S$. Freeze up jamming and formation of ice cover. In River ice Formation.Committee on River Ice Processes and the Environment, Canadian Geophysical Union, Hydrology Section: Edmonton, AB, Canada. 2013. P. 181-256.

6. Krupina N.A. et al. Comprehensive studies of the processes of growth and mechanics of destruction of ice of natural freezing in the large ice basin of the AARI. Problems of the Arctic and Antarctic. 2008. N 2. P. 7-20. (in Russian).

7. Buznik V.M. et al. Physical and mechanical properties of composite materials based on ice matrix. Materials Science. 2017. N 2. P. 33-40. (in Russian).

8. Vasiliev N.K., Ivanov A.A., Shatalina I.N. Methods of hardening and reinforcement of ice for structures of hydraulic engineering structures from ice and ice-ground composites. Siberian Journal of Pure and Applied Mathematics. 2013.V. 13. N 3. P. 31-37. (in Russian).

9. Herrnring H. et al. A cohesive model for ice and its verification with tensile splitting tests. 12th European LS-DYNA Conference 2019. Koblenz, Germany. 2019. 9 p. 
11. Гордон Д. и др. Конструкции, или почему не ломаются вещи. М.: Издательство Мир. 1980. 390 с.

12. Гончарова Г.Ю. и др. Исследование возможностей снижения фрикционного взаимодействия в паре "конек-лед" путем модификации структуры обеих контактирующих поверхностей. Вестник Российского фонда фундаментальных исследований. 2015. №. 3. С. 45-53.

13. Гончарова Г.Ю. и др. Химическое модифицирование льда и материалов на его основе с целью управления их свойствами. Химическая технология. 2020. Т. 21. №. 12. C. 548-560.

14. Wilson P. Recent developments in the study of recrystallization. Croatia: InTech, 2013. 232 p.

15. Kapembwa M., Rodríguez-Pascual M., Lewis A. Heat and mass transfer effects on ice growth mechanisms in pure water and aqueous solutions. Crystal Growth Design. 2014. N 14. P. 389-395. DOI: $10.1021 / \mathrm{cg} 401428$.

16. Каур И., Густ В. Диффузияпограницамзеренифаз: Пер. сангл. М.: 1991.448 с.

17. Patankar $S$. Numerical heat transfer and fluid flow. Boca Raton. 1980.214 p.
10. Iliescu D. et al. Strengtheningicethroughcyclicloading. Journal of Glaciology. 2017. V. 63. N 240. P. 663-669. DOI: 10.1017/jog.2017.32.

11. Gordon D.et al. Constructions, or why things don't break. M.: Publishing house Mir. 1980. 390 p. (in Russian).

12. Goncharova G. Yu. et al. Investigation of the possibilities of reducing the frictional interaction in a pair of "skate-ice" by modifying the structure of both contacting surfaces. Bulletin of the Russian Foundation for Basic Research. 2015. N 3. P. 45-53. (in Russian).

13. Goncharova G. Yu. et al. Chemical modification of ice and materials based on it to control their properties. Chemical Technology. 2020. V. 21. N 12/ P. 548-560. (in Russian).

14. Wilson P. Recent developments in the study of recrystallization. Croatia: InTech, 2013. 232 p.

15. Kapembwa M., Rodríguez-Pascual M., Lewis A. Heat and mass transfer effects on ice growth mechanisms in pure water and aqueous solutions. Crystal Growth Design. 2014. N 14. P. 389-395. DOI: $10.1021 / \mathrm{cg} 401428$.

16. Kaur I., Gust V. Diffusion along grain and phase boundaries: Transl. from English M.: 1991.448 p. (in Russian).

17. Patankar $S$. Numerical heat transfer and fluid flow. Boca Raton. 1980.214 p.

Поступила в редакичию (Received) 01.08.2021 Принята к опубликованию (Accepted) 03.09.2021 